\title{
Xuebijing injection reduces organ injuries and improves survival by attenuating inflammatory responses and endothelial injury in heatstroke mice
}

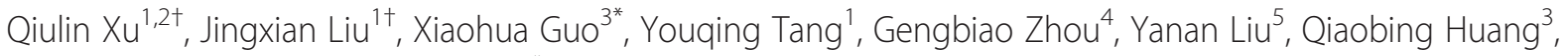
Yan Geng ${ }^{1}$, Zhifeng Liu ${ }^{1}$ and Lei Su ${ }^{1 *}$

\begin{abstract}
Background: The pathogenesis of heatstroke is a multi-factorial process involved with an interplay among subsequent inflammation, endothelial injury and coagulation disturbances, which makes pharmacological therapy of heatstroke a challenging problem. Xuebijing injection (XBJ), a traditional Chinese medicine used to sepsis, has been reported to suppress inflammatory responses and restore coagulation disturbances. However, little is known about the role of XBJ in heatstroke.
\end{abstract}

Methods: Mice were treated with indicated dose of XBJ before and/or after the induction of heatstroke. Serum inflammatory cytokines, tumor necrosis factor-a (TNF-a) and interleukin-6 (IL-6), and endothelial markers, von Willebrand Factor (VWF) and E-selectin, were measured by ELISA. Liver, kidney and heart profiles including alanine aminotransferase, aspartic aminotransferase, creatinine, blood urea nitrogen, and lactate dehydrogenase, were evaluated by UniCel DxC 800 Synchron Clinical Systems, and troponin was measured by ELISA. Coagulation profiles, including thrombin time, prothrombin time, activated partial thromboplastin time, international normalized ratio, and fibrinogen were examined by STA Compact ${ }^{\oplus}$ Hemostasis System. Jejunum injury was evaluated with H\&E staining. Changes in mitochondrial structure in cardiac tissue were assesed by electron microscopy.

Results: Pretreatment with XBJ decreased serum pro-inflammatory cytokines including TNF-a and IL-6, as well as endothelial injury markers, VWF and E-selectin, in a dose-dependent manner in heatstroke mice. Similar protective effects were observed when XBJ was administered after, or both before and after heat insult. These protective effects lasted for over $12 \mathrm{~h}$ in mice receiving XBJ before and after heat insult. XBJ also improved survival rates in heatstroke mice, ameliorated liver, heart, and kidney injuries, including mitochondrial damage to the heart, and reduced coagulation disturbances.

Conclusions: XBJ prevents organ injuries and improves survival in heatstroke mice by attenuating inflammatory responses and endothelial injury. XBJ may be a potentially useful in the prevention and treatment of heatstroke.

Keywords: Xuebijing injection, Heatstroke, Inflammatory responses, Endothelial injury

\footnotetext{
* Correspondence: lanblue97@gmail.com; slei_icu@126.com

${ }^{\dagger}$ Equal contributors

${ }^{3}$ Department of Pathophysiology, Southern Medical University, Key lab of

Shock and Microcirculation Research of Guangdong Province, Guangzhou

510515, China

'Department of ICU, General Hospital of Guangzhou Military Command, Key

Laboratory of Tropical Zone Trauma Care and Tissue Repair of PLA,

Guangzhou 510010, China

Full list of author information is available at the end of the article
}

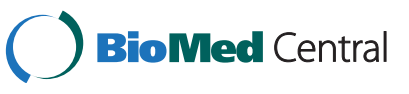

(C) 2015 Xu et al.; licensee BioMed Central. This is an Open Access article distributed under the terms of the Creative Commons Attribution License (http://creativecommons.org/licenses/by/4.0), which permits unrestricted use, distribution, and reproduction in any medium, provided the original work is properly credited. The Creative Commons Public Domain Dedication waiver (http://creativecommons.org/publicdomain/zero/1.0/) applies to the data made available in this article, unless otherwise stated. 


\section{Background}

Heatstroke, a potentially fatal condition occurring during summer heat waves, is characterized by core body temperature as high as $42^{\circ} \mathrm{C}$ accompanied by central nervous system dysfunction. Despite advances in prevention and treatment, the mortality rate from heatstroke remains high, varying from $10 \%$ to $50 \%$ [1]. Severe heatstroke is often accompanied by multiple organ dysfunction syndrome (MODS), which is resulted from the complex interplay among endothelial injury, inflammation, and coagulation responses of the host following the direct cytotoxic effects of heat [2]. Heat stress increases vascular permeability, promotes a pro-thrombotic state, and enhances the in vitro expression of adhesion molecules in endothelium. Markedly elevation in the concentrations of circulating inflammatory cytokines, including interleukin-6 (IL-6), tumor necrosis factor- $\alpha$ (TNF- $\alpha$ ), and interleukin- $1 \beta$ (IL-1 $\beta$ ), have been observed and histological examination has shown widespread hemorrhage, thrombosis, and migration of leukocytes both in animals and patients with severe heatstroke [3-7]. This constellation of events alters blood flow in the microcirculation, resulting in injuries to the endothelium and tissues. Although several drugs have been found to reduce inflammatory responses and improve prognosis in animals with heatstroke [8-13], none has been shown to reduce mortality in patients with heatstroke.

Xuebijing (XBJ) is a traditional Chinese combination medicine, which has been approved by the State Food and Drug Administration of China for the treatment of sepsis. XBJ consists mainly of extracts from five Chinese herbals: Carthamus tinctorius L., Paeonia lacti-flora Pall, Ligusticum chuanxiong hort, Salvia miltiorrhiza Bunge, and Angelica sinensis. Injected XBJ has been widely used to treat sepsis-related illnesses, including acute liver and kidney injury and autoimmune diseases [14-16]. XBJ was also found to reduce circulating TNF- $\alpha$, IL- 6 , IL-1 $\beta$, and IL-8 $[17,18]$, to maintain immunologic balance by correcting disorders of T-lymphocyte subpopulations, and to alleviate endotoxin-induced disseminated intravascular coagulation in rabbits [19]. We recently reported that XBJ alleviated liver injury in heatstroke rats [16]. However, the role and underlying mechanisms of XBJ in heatstroke remain to be determined. This study was designed to assess the role of XBJ in the release of inflammatory cytokines, in coagulation abnormalities and in endothelial injury in heatstroke mice.

\section{Methods}

\section{Chemicals and reagents}

XBJ, consisting of a 1:1:1:1:1 mixture of extracts of five herbs, Carthamus tinctorius L., Paeonia lacti-flora Pall, Ligusticum chuanxiong hort, Salvia miltiorrhiza Bunge, and Angelica sinensis, was supplied by Tianjin Chase
Sun Pharmaceutical Co., Ltd (Tianjin, China; batch number of 1303031), and stored at room temperature.

\section{Animals treatments}

All protocols were approved by the Animal Ethics Committee of the General Hospital of Guangzhou Military Command, in accordance with the Guide for the Care and Use of Laboratory Animals of the National Institutes of Health. Male C57BL/6 J mice, aged 10-12 weeks and weighing 20-25 g, were obtained from the Animal Resource Center of Southern Medical University and maintained at $23 \pm 1^{\circ} \mathrm{C}$ with a 12-hour light/dark cycle. Animals were randomized into three groups, a sham-heated group (Sham), a heatstroke group (HS) and a heatstroke plus XBJ group (HSXBJ). Four animals in each group were assessed for biomarkers at each indicated time point, with survival assessed in eight mice in the Sham group and 22 each in the HS and HSXBJ groups. Animals were intraperitoneally injected with $0.5 \mathrm{ml}$ of $0.9 \%$ saline or the indicated doses of $\mathrm{XBJ}$ dilutions at the indicated times. XBJ doses were based on the doses used in patients with sepsis, with $1 \mathrm{ml} / \mathrm{kg}$ used to treat moderate sepsis and $2 \mathrm{ml} / \mathrm{kg}$ used to treat severe sepsis plus MODS. Heatstroke was induced by placing the animals in an artificial climate chamber at $38 \pm 0.5^{\circ} \mathrm{C}$ and a relative humidity of $60 \% \pm 5 \%$. Rectal temperature (Tc) was measured every $10 \mathrm{~min}$ by a thermometer. When Tc reached $42.7^{\circ} \mathrm{C}$, the criterion for heatstroke onset, the animals were removed from the chamber and allowed to recover at room temperature $\left(24 \pm 2^{\circ} \mathrm{C}\right)$. Animals in the Sham group were treated identically to those in the HS group, except that artificial climate chamber was maintained at $24 \pm 0.5^{\circ} \mathrm{C}$. Animals were anesthetized by intraperitoneal injection of sodium pentobarbital $(50 \mathrm{mg} / \mathrm{kg}$ body weight) and sacrificed at the indicated times after heatstroke onset. Bloood and organ samples were then collected. Serum concentrations of cytokines and biochemical markers, as well as histological and ultramicrostructural changes, were assessed in animals sacrificed $6 \mathrm{~h}$ after heatstroke onset. To determine the time-course of serum IL-6, TNF- $\alpha$, vWF, and E-selectin concentrations after heatstroke induction, animals were sacrificed at 0,2 , $4,6,8,10$, and $12 \mathrm{~h}$ after heatstroke induction. Animals used to evaluate survival rate were from separated group and were not sacrificed until $72 \mathrm{~h}$ of observation.

\section{Enzyme-linked immunosorbent assay (ELISA)}

Blood samples were collected by cardiac puncture, maintained at room temperature for $30 \mathrm{~min}$, and centrifuged at $1300 \times \mathrm{g}$ for $15 \mathrm{~min}$ to obtain serum. Serum samples were stored in aliquots at $-80^{\circ} \mathrm{C}$ until assayed. The serum concentrations of TNF- $\alpha$, IL-6, von Willebrand Factor antigen (vWF:Ag) (Ramco Laboratory) and E-selectin were evaluated by ELISA ( $\mathrm{R} \& \mathrm{D}$ Systems) according to the manufacturer's instructions . 


\section{Organ injuries}

Liver, kidney and heart profiles, including alanine aminotransferase (ALT), aspartic aminotransferase (AST), creatinine $(\mathrm{Cr})$, blood urea nitrogen (BUN), and lactate dehydrogenase (LDH) levels, were evaluated by UniCel DxC 800 Synchron Clinical Systems (United States), and troponin (Tn) concentration was determined by ELISA (MyBioSource, United States). Coagulation profiles including thrombin time (TT), prothrombin time (PT), activated partial thromboplastin time (APTT), international normalized ratio (INR), and fibrinogen concentration, were assessed using an STA Compact ${ }^{\circ}$ Hemostasis System (France).

\section{Histological examination}

Tissue samples from jejunum were obtained $6 \mathrm{~h}$ after heatstroke onset, fixed in $4 \%$ paraformaldehyde, embedded in paraffin, cut into $5-\mu \mathrm{m}$ sections with a microtome, and stained with hematoxylin and eosin. Stained specimens were examined by light microscopy.

\section{Electron microscopy}

Cardiac tissue was quickly cut into 1 -mm cubes, incubated overnight at $4^{\circ} \mathrm{C}$ in $2.5 \%$ glutaraldehyde in $0.1 \mathrm{~mol} / 1$ phosphate buffer ( $\mathrm{pH}$ 7.4), and fixed in 1\% buffered osmium tetroxide. The specimens were conventionally processed and evaluated by electron microscopy (H-800; Hitachi).

\section{Statistical analysis}

All continuous data were expressed as mean $\pm \mathrm{SE}$ and compared by one-way analysis of variance with Fisher's LSD test. Survival curves were plotted by the KaplanMeier method and compared by the log-rank test. P $<0.05$ was considered statistically significant. All statistical analyses of data were performed using SPSS 16.0 (SPSS Inc., Chicago, IL).

\section{Results}

\section{Dose-dependent effects of XBJ on inflammation and} endothelial injury

E-selectin and vWF, regarded as biomarkers of endothelial injury, and IL- 6 and TNF- $\alpha$, key factors in systemic inflammatory responses, have been reported to be increased in heatstroke [1,3]. To determine the role of $\mathrm{XBJ}$ in heatstroke-induced inflammatory responses and endothelial injury, we assessed whether XBJ affects serum levels of these four proteins in heatstroke. Mice were treated with 2,4 , or $10 \mathrm{ml} / \mathrm{kg}$ of XBJ before heat insult, and serum concentrations of IL-6, TNF- $\alpha$, E-selectin and vWF were measured $6 \mathrm{~h}$ after heatstroke onset. The concentrations of all four proteins were higher in the HS than in the Sham group. XBJ dose-dependently reduced the serum concentrations of these four proteins, with $10 \mathrm{mg} / \mathrm{kg}$ of XBJ reducing serum IL- 6 and TNF- $\alpha$ about
80\% each and serum E-selectin and vWF about 50\% each (Figure 1A-D). As $2 \mathrm{ml} / \mathrm{kg}$ of XBJ is used to treat septic patients with MODS, further experiments assessed the effects of $4 \mathrm{ml} / \mathrm{kg}$ of XBJ, thus balancing experimental effects and clinical significance.

\section{Effects of time of XBJ administration on inflammation and endothelial injury}

To investigate whether the effects of XBJ administered after heatstroke onset were similar to those of XBJ administered before heat insult, mice were treated with the same dose of XBJ before or after heatstroke. Interestingly, we found the XBJ-induced reductions in IL-6, E-selectin and vWF concentrations were similar in mice treated with XBJ before heat insult or after heatstroke onset, whereas serum TNF- $\alpha$ was lower in in mice treated with XBJ before heat insult than after heatstroke onset. As a traditional Chinese medicine compound, the effective duration of $\mathrm{XBJ}$ is difficult to determine and not reported. The recommended time interval of consecutive uses of XBJ is usually $12 \mathrm{~h}$, and 6 to $8 \mathrm{~h}$ for critical patients according to its clinical instructions. In studies with human or animals, XBJ is usually used 1 to 3 times per day. In this set, we administered mice a second dose of XBJ after heatstroke since the experiment spanned 5-h of heat insult and 6-h of observation. However, the second dose of XBJ did not result in additional decreases in these four proteins compared with the same dose of saline given after heatstroke, but did reduce serum concentrations of IL-6, TNF- $\alpha$, and E-selectin (Figure 2A-D). These findings suggested that XBJ attenuated inflammatory responses and endothelial injury when given after heatstroke.

\section{Influences of XBJ on time course of inflammatory and endothelial biomarkers}

To further assess the effects of $\mathrm{XBJ}$ on pro-inflammatory cytokines and markers of endothelial injury, we analyzed the influences of XBJ on the kinetics of these biomarkers. Mice were pretreated with $\mathrm{XBJ}(4 \mathrm{ml} / \mathrm{kg})$ and serum concentrations of IL- 6 , TNF- $\alpha$, E-selectin, and vWF were assayed 2, 4, 6, 8, 10, and $12 \mathrm{~h}$ after heatstroke. We found that the elevations in all four proteins observed for $12 \mathrm{~h}$ after heatstroke were attenuated by XBJ pretreatment, suggesting that the protective role of XBJ in heatstroke was due to its attenuation of circulating pro-inflammatory cytokines and markers of endothelial injury (Figure 3A-D).

\section{Effects of XBJ on organ injuries}

To assess the effects of XBJ on organ injuries, mice were sacrificed $6 \mathrm{~h}$ after heatstroke onset, and markers of liver, heart and kidney injury, as well as histological changes in the jejunum, were examined. Liver, heart and kidney injuries in heatstroke mice, characterized by markedly elevated serum concentrations of ALT, AST, Cr, BUN, LDH and 


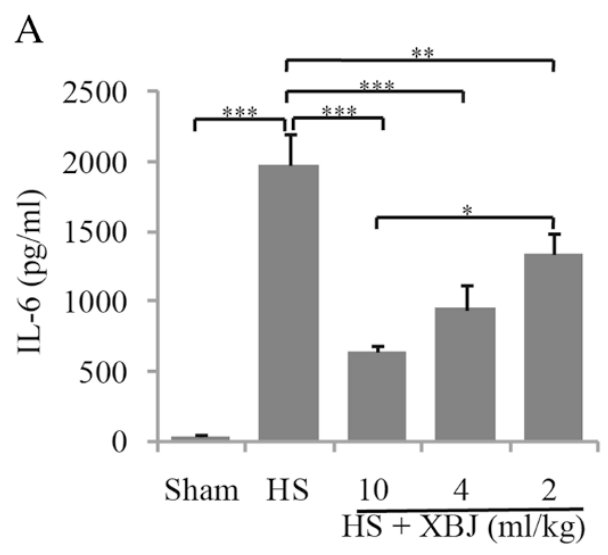

$\mathrm{C}$

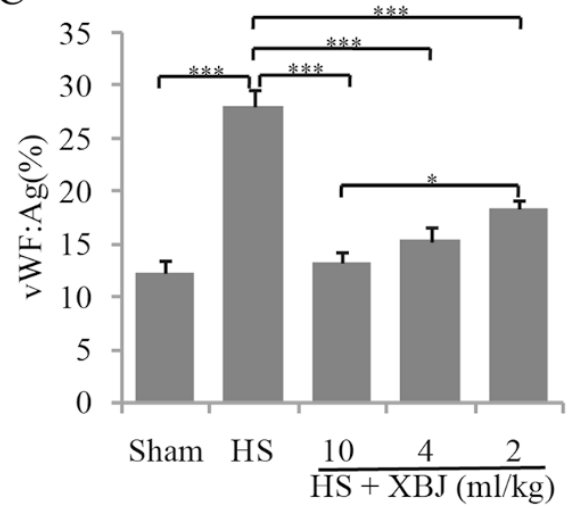

B

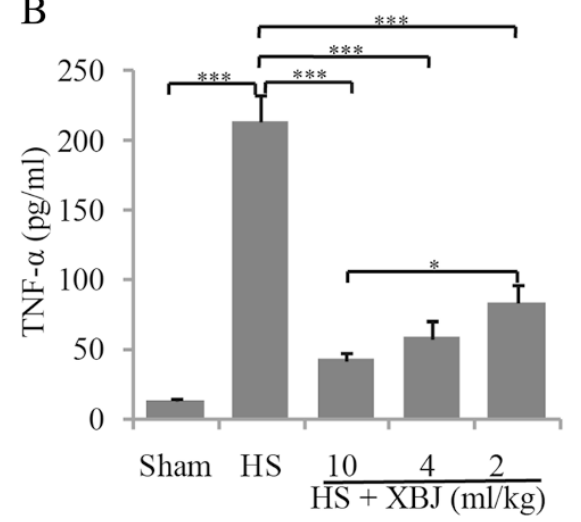

D

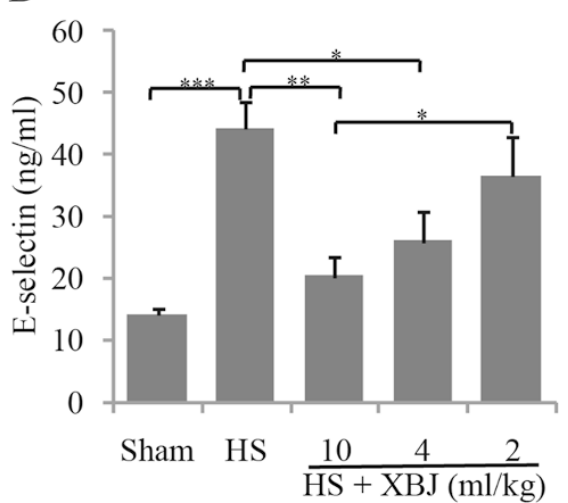

Figure 1 Dose-dependent effects of XBJ on serum IL-6, TNF-a, vWF and E-selectin. Mice were treated with $0.9 \%$ saline or 2, 4, or $10 \mathrm{ml} / \mathrm{kg}$ of XBJ, followed by heat insult. Serum IL-6 (A), TNF-a (B), VWF (C), and E-selectin (D) levels were determined with ELISA $6 \mathrm{~h}$ after heatstroke onset. ${ }^{*} \mathrm{P}<0.05$, ${ }^{* *} \mathrm{P}<0.01$, ***P $<0.001$.

Tn, were attenuated by XBJ (Table 1). Heatstroke-induced injury to the jejunum, characterized by dramatic shortened villi, disrupted integrity of the intestinal epithelial layer and complete loss of architecture of the lamina propria and submucosa, was diminished by XBJ (Figure 4A).

\section{Effects of XBJ on coagulation disturbance}

Coagulation disturbance is a marked feature and a leading cause of heatstroke. We evaluated the influences of $\mathrm{XBJ}$ on coagulation, and found that HS mice had a significantly prolonged PT, increased INR and elevated serum fibrinogen compared with those in the Sham group. All of these abnormalities, however, were prevented by $\mathrm{XBJ}$ (Figure 5A-E).

\section{Effects of XBJ on mitochondrial structure}

As shown by transmission electron microscopy, heat stress caused uniform mitochondrial swelling in the heart, with some mitochondria also showing disorganized cristae, decreased matrix density, and emerging amorphous matrix densities or granular dense bodies. These ultrastructural changes in the mitochondria were diminished by XBJ (Figure 4B).

\section{Effects of XBJ on mouse survival}

As our preliminary study showed that no animal died over the $72 \mathrm{~h}$ after heatstroke onset, animals in each group were monitored for $72 \mathrm{~h}$ to determine their survival rates. All animals in Sham group remained alive, whereas 16 of $22(73 \%)$ in the HS group, and 10 of $22(45 \%)$ in the HSXBJ group died (Figure 6). The survival rate was therefore $38 \%$ higher in the HSXBJ than in the HS group $(\mathrm{P}=0.006)$.

\section{Discussion}

Although efforts have been made to develop new methods to treat heatstroke during last decades, few advances have been achieved in early management, except for rapid cooling and prompt fluid resuscitation. Although several drugs have been investigated in the treatment of heatstroke in animals, however, as we know, they are still challenged in patients. XBJ has been found to be effective and safe for the treatment of sepsis and sepsis-related diseases. Most recently, XBJ pretreatment before heat insult was found to reduce plasma concentrations of IL- 6 and TNF- $\alpha$ and to decrease organ injuries in rats [20]. However, it is unlikely to administer XBJ before heatstroke in clinical use and 

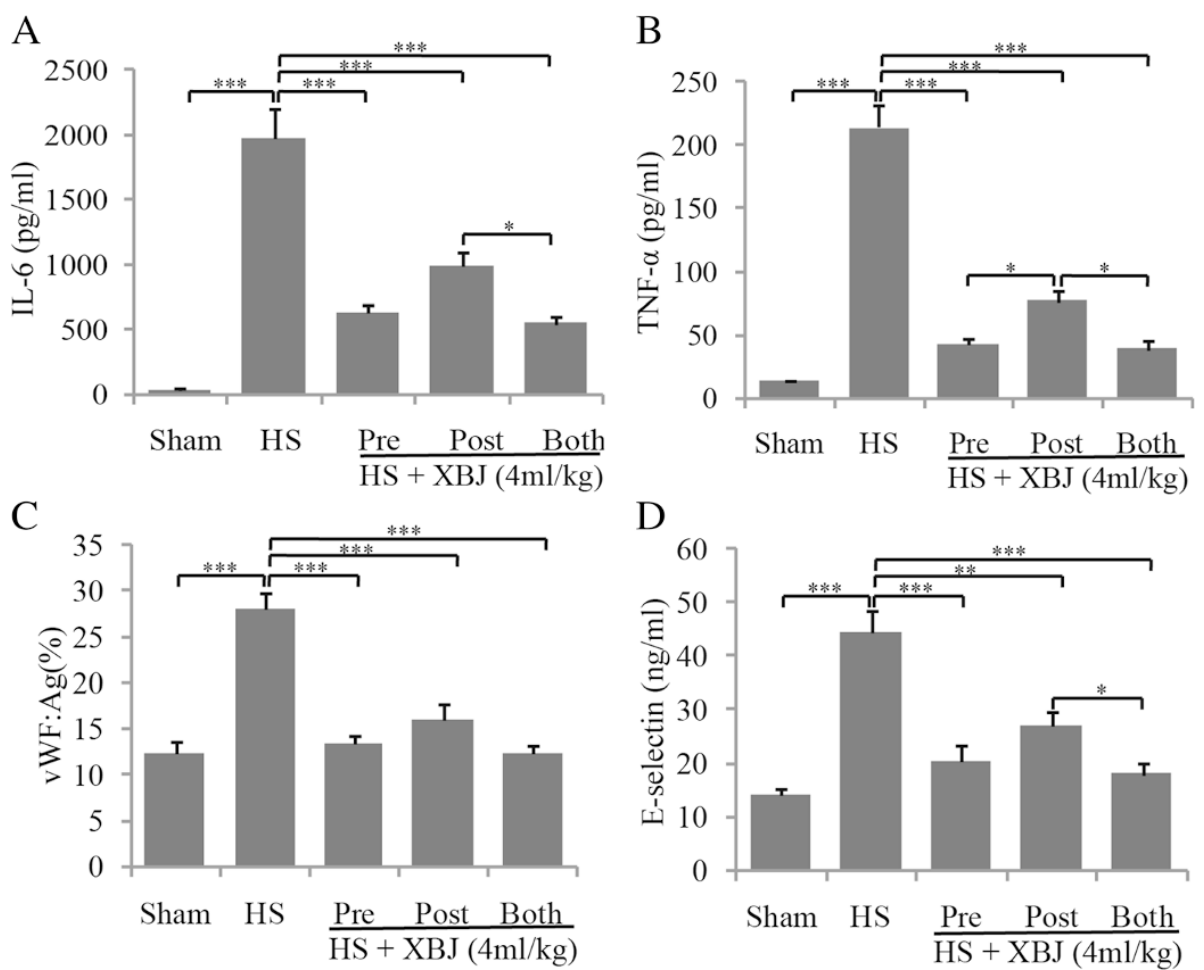

Figure 2 Effects of administration time of XBJ on serum IL-6, TNF-a, vWF and E-selectin. Mice subjected to heat stress were treated with $0.9 \%$ saline or $4 \mathrm{ml} / \mathrm{kg}$ of XBJ 30 min before heat insults (Pre), post heatstroke onset (Post), or both before heat insults and post heatstroke onset (Both). Serum IL-6 (A), TNF-a (B), VWF (C), and E-selectin (D) levels were determined with ELISA $6 \mathrm{~h}$ after heatstroke onset. ${ }^{*} P<0.05$, ${ }^{* *} P<0.01$, ${ }^{* * *} P<0.001$.
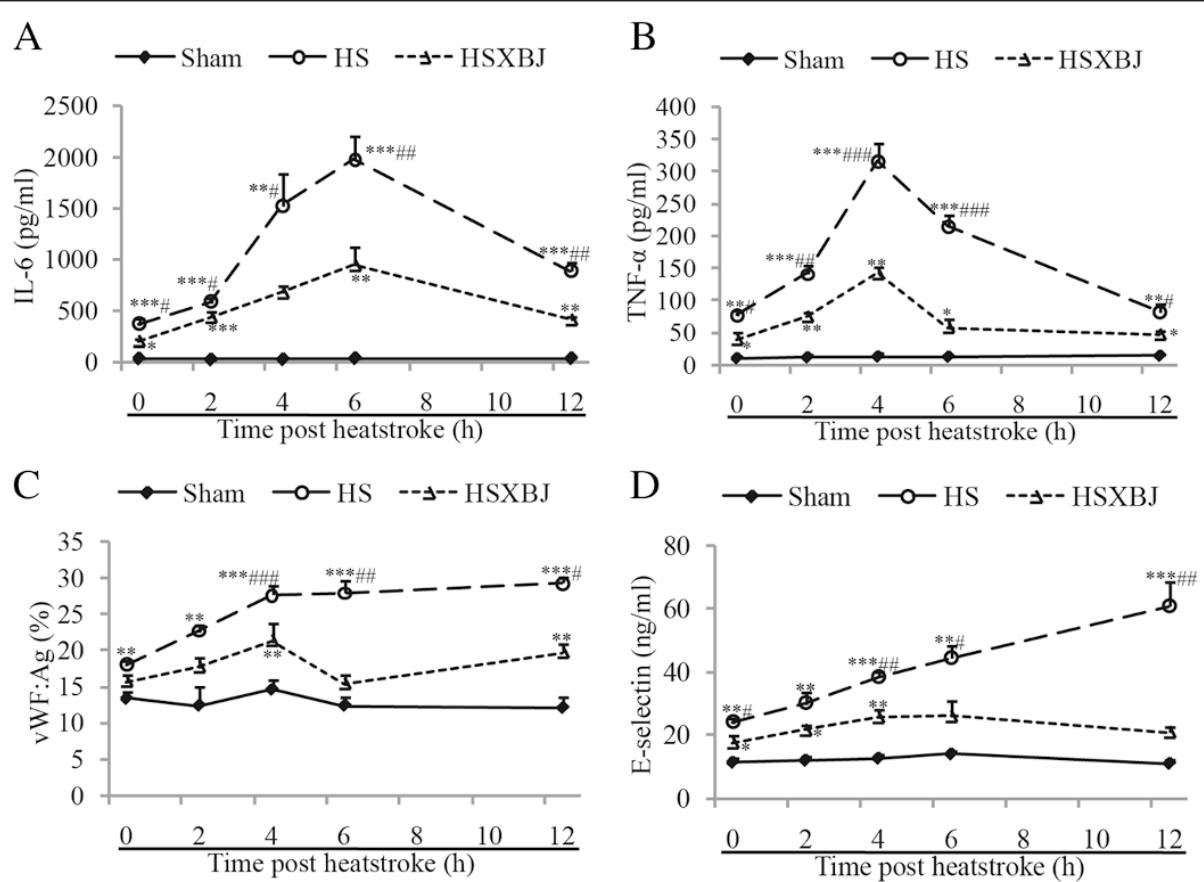

Figure 3 Time course of serum IL-6, TNF-a, vWF, and E-selectin after heatstroke induction. Mice were treated with $0.9 \%$ saline (HS) or $4 \mathrm{ml} / \mathrm{kg}$ of XBJ (HSXBJ), followed by heat insult. Serum IL-6 (A), TNF-a (B), VWF (C), and E-selectin (D) levels were determined with ELISA at 0, 2, 4, 6, and 12 h after heatstroke onset. ${ }^{*} \mathrm{P}<0.05$, ${ }^{* *} \mathrm{P}<0.01$, ${ }^{* * *} \mathrm{P}<0.001$, vs. sham group; ${ }^{*} \mathrm{P}<0.05$, ${ }^{\# \#} \mathrm{P}<0.01$, ${ }^{\# \# \#} \mathrm{P}<0.001$, vs. HSXBJ group. 
Table 1 Biochemical markers of tissue and organ injury in mice subjected to heatstroke

\begin{tabular}{llll}
\hline & Sham & HS & HSXBJ \\
\hline ALT $(\mathrm{U} / \mathrm{L})$ & $27.0 \pm 4.2$ & $283.3 \pm 37.9^{* * *}$ & $71.3 \pm 10.2^{\# \# \#}$ \\
AST $(\mathrm{U} / \mathrm{L})$ & $21.5 \pm 5.4$ & $426.3 \pm 47.1^{* * *}$ & $162.5 \pm 24.0^{\# \# \#}$ \\
$\mathrm{Cr}(\mu \mathrm{mol} / \mathrm{L})$ & $32.8 \pm 6.0$ & $172.0 \pm 17.7^{* * *}$ & $116.3 \pm 11.5^{\#}$ \\
$\mathrm{BUN}(\mathrm{mmol} / \mathrm{L})$ & $2.9 \pm 0.6$ & $18.7 \pm 1.4^{* * *}$ & $13.2 \pm 1.1^{\# \#}$ \\
$\mathrm{LDH}(\mathrm{U} / \mathrm{L})$ & $81.0 \pm 9.6$ & $439.0 \pm 62.7^{* * *}$ & $165.3 \pm 22.2^{\# \# \#}$ \\
$\mathrm{Tn}(\mu \mathrm{g} / \mathrm{L})$ & $0.12 \pm 0.02$ & $4.08 \pm 0.59^{* * *}$ & $1.65 \pm 0.31^{\# \#}$ \\
\hline
\end{tabular}

Mice pretreated with $4 \mathrm{ml} / \mathrm{kg}$ of XBJ (HSXBJ group) or $0.9 \%$ saline (HS group) were subjected to heat insults. Values represents mean $\pm \mathrm{SE}$. ALT, alanine aminotransferase; AST, aspartic aminotransferase; $\mathrm{Cr}$ creatinine; BUN, blood urea nitrogen, $\mathrm{LDH}$, lactate dehydrogenase; Tn, troponin. ${ }^{* * *} \mathrm{P}<0.001$, vs. sham group; ${ }^{\#} \mathrm{P}<0.05,{ }^{\# \#} \mathrm{P}<0.01$, ${ }^{\# \#} \mathrm{P}<0.001$, vs. HS group.

since human heatstroke is an emergency condition, which rapidly progresses to MODS and death, it is necessary to assess the effect of XBJ administered after heatstroke onset. Moreover, as a combined traditional Chinese medicine, XBJ possesses other therapeutic effects beyond its anti-inflammation role, which remain to be elucidated. This study showed that XBJ reduced the circulating inflammatory cytokines and markers of endothelial injury, attenuated injuries to the liver, heart and kidneys, restored coagulation balance, and improved survival. Importantly, we found that administration of XBJ before and after heatstroke onset had similar effects. Moreover, we found that XBJ prevents heat stress-induced mitochondrial damage to heart.

Systemic inflammatory response is an important cause of multiple organ injuries in heatstroke. Increased circulating inflammatory cytokines, including IL-6, TNF- $\alpha$, IL- 8 and IL- $1 \beta$, are commonly observed in animals and patients with heatstroke and high levels of these proteins are correlated with morbidity and mortality $[1,11,21,22]$. Inhibition of systemic inflammatory responses by electrical vagus nerve stimulation [1], administration of IL-1 receptor antagonist [23], glucocorticoids [24], recombinant activated protein $C[9,25]$, recombinant thrombomodulin [26], or antithrombin [27], or knockout of the gene encoding Toll-like receptor 4, a central regulator of innate immune responses [21], has been found to prevent organ damage and to improve survival. In this study, we demonstrated that XBJ dose-dependently reduced serum IL-6 and TNF- $\alpha$ in heatstroke mice and these anti-inflammation effects lasted for over $12 \mathrm{~h}$. Moreover, XBJ administered before heat insult and after heatstroke onset had comparable anti-inflammation effects.

The mechanism by which XBJ suppresses inflammation remains to be determined. Loss of intestine barrier function is regarded as a fuel of innate SIRS. Prolonged heat exposure decreases splanchnic blood flow and increases

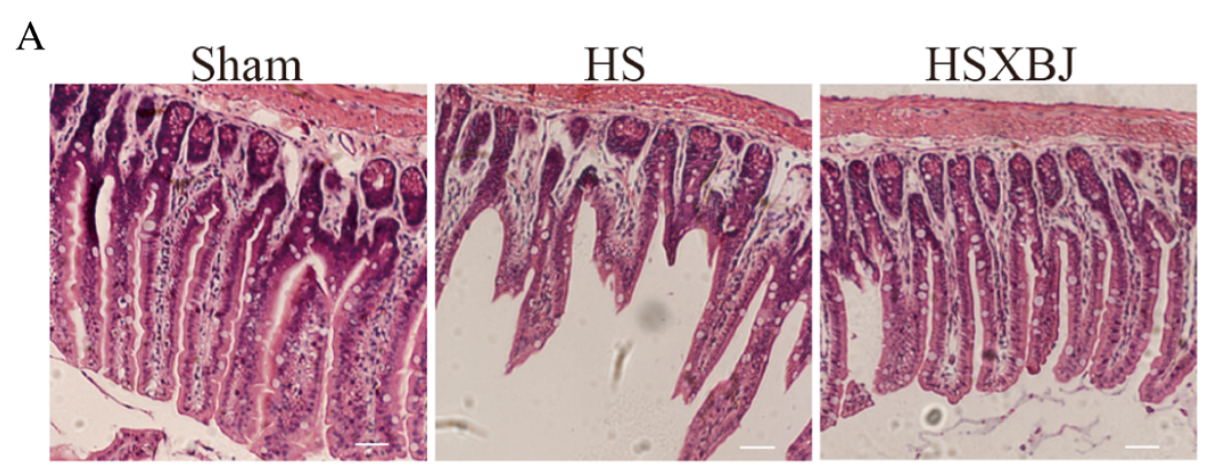

B

Sham

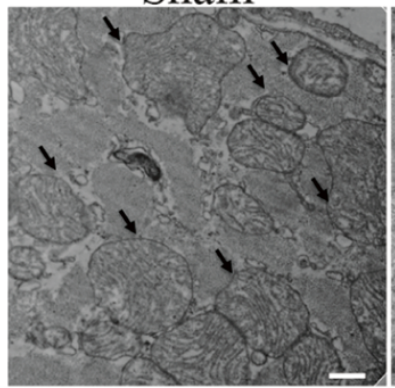

HS

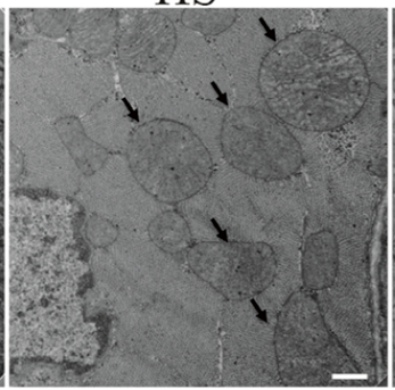

HSXBJ

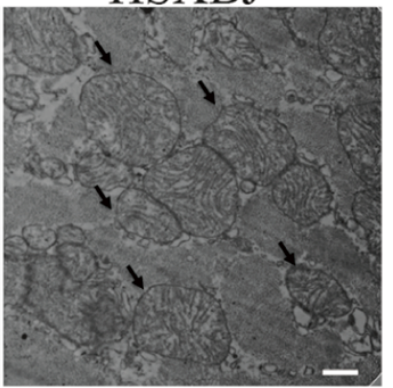

Figure 4 Histological changes in the jejunum and heart mitochondrial ultrastructure. Mice were treated with $4 \mathrm{ml} / \mathrm{kg}$ of XBJ (HSXBJ group) or $0.9 \%$ saline (HS group), followed by heat insults. (A) Tissue samples from jejunum were stained with H\&E. Scale bar, $10 \mu \mathrm{m}$. (B) Representative electron micrographs of the heart showing mitochondrial structure (arrows). Scale bar, $500 \mathrm{~nm}$. 

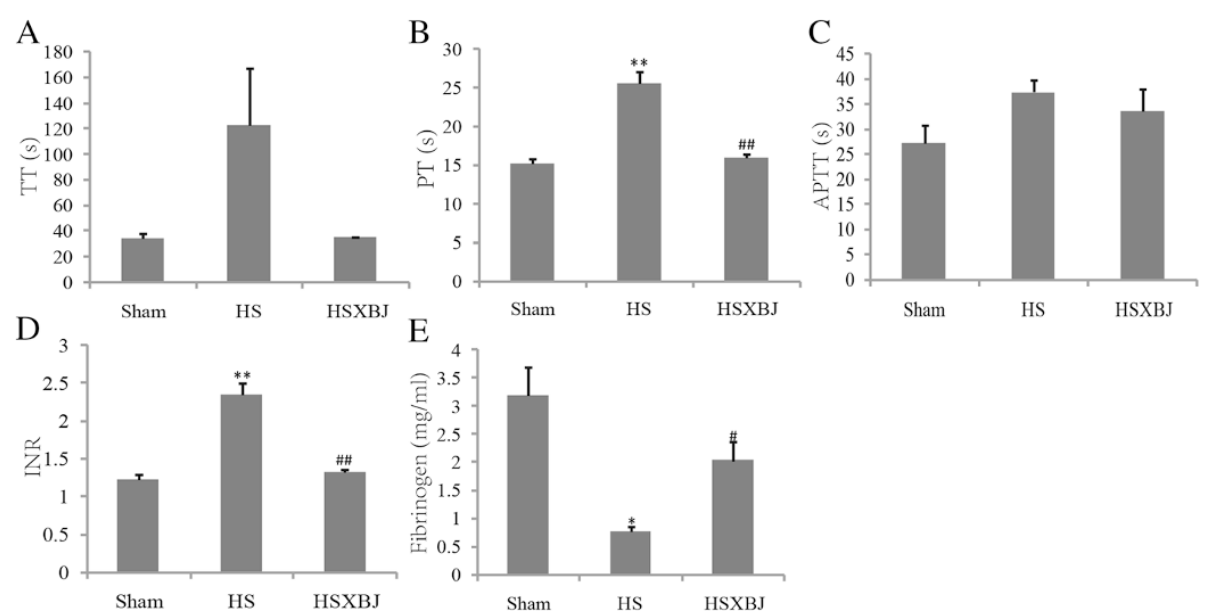

Figure 5 Changes in coagulation in heatstroke mice. Mice pretreated with $4 \mathrm{ml} / \mathrm{kg}$ of XBJ (HSXBJ group) or $0.9 \%$ saline (HS group) were subjected to heat insults. Coagulation was evaluated by measuring (A) TT, (B) PT, (C) APTT, (D) INR, and (E) fibrinogen. *P $<0.05$, **P $<0.001$, vs Sham; ${ }^{\# P}<0.05,{ }^{\# \#} P<0.001$, vs HS group. $T$ T, thrombin time; PT, prothrombin time; APTT, activated partial thromboplastin time; INR, international normalized ratio.

nitrosative and oxidative stress, resulting in disruption of tight junctions, loss of intestine barrier function and intestinal hyper-permeability. These, in turn, lead to the translocation into systemic circulation of bacteria and endotoxins normally contained in the gut lumen [28]. In this study, histological examination showed that XBJ maintained the integrity of intestinal barrier structure, preventing the translocation of bacteria and endotoxins and possibly contributing to reduced systemic inflammatory responses. The anti-inflammation activity of XBJ may also be linked to NF-kB, a key transcription factor that controls the induction of pro-inflammatory gene expression and has attracted interest as a new target for the treatment of inflammatory diseases. Recently, some active ingredients in XBJ, including senkyunolide I, safflor yellow
A, oxypaeoniflorin, and benzoylpaeoniflorin, were identified to inhibit NF-kB activity by a bio-activity-integrated ultra-performance liquid chromatography quadrupole time-of-flight mass spectrometry assay system [17], indicating that the anti-inflammation activity of XBJ involves inhibition of the NF-kB pathway. Moreover, Highmobility group box 1 (HMGB1), a cytokine mediator of lethal systemic inflammation in sepsis and non-sepsis diseases, has been implicated in heatstroke-induced SIRS and direct inhibition of HMGB1 with its monoclonal antibody can decrease circulating inflammatory cytokines [29]. XBJ can inhibit HMGB1 expression in some sepsis related diseases $[14,30]$, suggesting that XBJ may attenuate inflammatory response partially by down-regulation of HMGB1 expression in heatstroke.

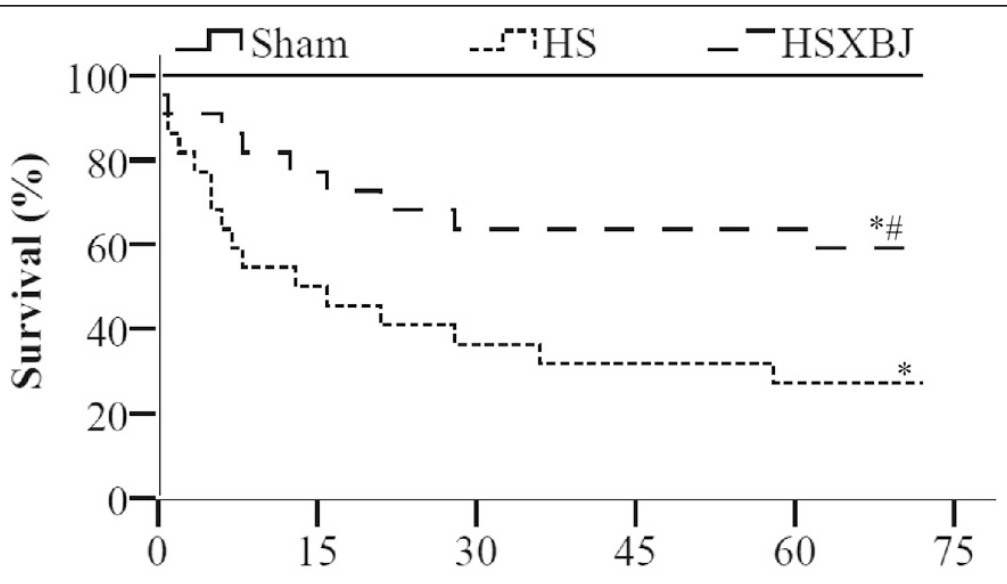

Time post heatstroke (h)

Figure 6 Effects of XBJ on mouse survival rate. Mice were pretreated with $4 \mathrm{ml} / \mathrm{kg}$ of XBJ (HSXBJ) or $0.9 \%$ saline (HS group), followed by heats insult. ${ }^{P}<<0.05$, vs Sham; ${ }^{\#} P<0.05$, vs HS group. sham: $n=8$; HS: $n=22 ; H S X B J: n=22$. 
Importantly, our data showed that XBJ reduced serum vWF and E-selectin in heatstroke mice, suggesting a protective role for $\mathrm{XBJ}$ in preventing endothelial injury. Endothelium regulates vascular tone, controls leukocyte recruitment, and maintains a balance between procoagulant and anticoagulant substances [31]. In heatstroke, widespread microvascular injury, thrombosis and inflammation resulted mainly from endothelial injury contributes to the development of MODS [2,3]. Both vWF and E-selectin synthesized in endothelial cells are elevated in response to inflammation and other stimulations and usually used as molecular markers of endothelial injury. vWF plays a crucial role in platelet adhesion and aggregation, the main initial steps in haemostasis after vascular injury, while E-selectin is important in mediating leukocyte trans-endothelial migration. Moreover, given that cross-talk between endothelial injury, coagulation and inflammation lead to the amplification and exacerbation of inflammatory responses, reduced endothelial injury contributes to ameliorated coagulation disturbances and systemic inflammatory responses in this study. Taken together, our results suggest that XBJ may ameliorate the effects of heatstroke by ablating endothelial injury.

Interestingly, we found that XBJ treatment reduced mitochondrial damages in heatstroke mice. Mitochondria are not only the site of energy production, but also a central locus in the regulation of cell death. Damage to mitochondrial structure generates large amounts of reactive oxygen species (ROS), and triggers cell death pathways of necrosis and apoptosis, which contribute to cell death and tissue injury $[32,33]$. Therefore, preservation of normal mitochondrial structure is an important cellular mechanism by which XBJ reduces organ injury resulting from heatstroke.

Strikingly, we found that XBJ had similar inhibitory effects on inflammatory responses and endothelial injury when it was administrated before heat insult or immediately after heatstroke onset, with these effects lasting for over $12 \mathrm{~h}$. These findings suggest that XBJ may be used in both the prevention and treatment of heatstroke. However, the effects of delayed administration of XBJ after heatstroke require further investigation.

\section{Conclusions}

In this study, we demonstrated that XBJ reduced organ injury and improved survival in heatstroke mice by attenuating systemic inflammatory responses and endothelial injury, suggesting the potential of $\mathrm{XBJ}$ in the prevention and treatment of patients with heatstroke. Although this study was limited to a mouse model and requires confirmation, it provided valuable insights into the role of XBJ in heatstroke. Several other herbal medicines containing anti-inflammation constituents, such as buckwheat sprouts and anti-asthma herbal medicine intervention $[34,35]$, may also protect against heatstroke. It is interesting to evaluate their roles in heatstroke in further studies.

\section{Abbreviations}

XBJ: Xuebijing injuection; VWF: Willebrand factor; IL-6: Interleukin - 6; TNFa: Tumor necrosis factor - $\alpha$; IL-1 $\beta$ : Interleukin - 1 $\beta$; ALT: Alanine aminotransferase; AST: Aspartic aminotransferase; Cr: Creatinine; BUN: Blood urea nitrogen; LDH: Actate dehydrogenase; Tn: Troponin; TT: Thrombin time; PT: Prothrombin time; APTT: Activated partial thromboplastin time; INR: International normalized ratio; ELISA: Enzyme-linked immunosorbent assay.

\section{Competing interests}

The authors declare that they have no competing interests.

\section{Authors' contributions}

Conceived and designed the experiments: $X G$ and LS. Performed the experiments: QX, JL, YT, GZ and YL; Analyzed the data: YG and ZL; Wrote the manuscript: QX and XG; Reviewed paper: LS. All authors read and approved the final manuscript.

\section{Acknowledgements}

This work was supported by grants from the PhD Start-up Fund of Natural Science Foundation of Guangdong Province, China (\$2013040015661), China Postdoctoral Science Foundation (2014 M552180), National Natural Science Foundation of China (31300950), and Project of Medical Research of PLA (BWS12J108).

\section{Author details}

'Department of ICU, General Hospital of Guangzhou Military Command, Key Laboratory of Tropical Zone Trauma Care and Tissue Repair of PLA, Guangzhou 510010, China. ${ }^{2}$ Postdoctoral Workstation, Huabo

Bio-pharmaceutical Research Institute, Guangzhou 510515, China. ${ }^{3}$ Department of Pathophysiology, Southern Medical University, Key lab of Shock and Microcirculation Research of Guangdong Province, Guangzhou 510515, China. ${ }^{4}$ Guangzhou University of Chinese Medicine, Guangzhou 510405, China. ${ }^{5}$ Southern Medical University, Guangzhou 510515, China.

Received: 26 August 2014 Accepted: 13 January 2015

Published online: 05 February 2015

\section{References}

1. Yamakawa K, Matsumoto N, Imamura Y, Muroya T, Yamada T, Nakagawa J, et al. Electrical vagus nerve stimulation attenuates systemic inflammation and improves survival in a rat heatstroke model. PLoS One. 2013;8(2):e56728.

2. Bouchama A, Knochel JP. Heat stroke. N Engl J Med. 2002;346(25):1978-88.

3. Roberts GT, Ghebeh H, Chishti MA, Al-Mohanna F, El-Sayed R, Bouchama A. Microvascular injury, thrombosis, inflammation, and apoptosis in the pathogenesis of heatstroke: a study in baboon model. Arterioscler Thromb Vasc Biol. 2008;28(6):1130-6.

4. al-Mashhadani SA, Gader AG, al Harthi SS, Kangav D, Shaheen FA, Bogus F. The coagulopathy of heat stroke: alterations in coagulation and fibrinolysis in heat stroke patients during the pilgrimage (Haj) to Makkah. Blood Coagul Fibrinolysis. 1994;5(5):731-6.

5. el-Kassimi FA, Al-Mashhadani S, Abdullah AK, Akhtar J. Adult respiratory distress syndrome and isseminated intravascular coagulation complicating heat stroke. Chest. 1986;90(4):571-4.

6. Bouchama A, Roberts G, Al Mohanna F, El-Sayed R, Lach B, Chollet-Martin S, et al. Inflammatory, hemostatic, and clinical changes in a baboon experimental model for heatstroke. J Appl Physiol. 2005;98(2):697-705.

7. Leon LR, Helwig BG. Heat stroke: role of the systemic inflammatory response. J Appl Physiol. 2010;109(6):1980-8.

8. Hagiwara S, Iwasaka H, Goto K, Ochi Y, Mizunaga S, Saikawa T, et al. Recombinant thrombomodulin prevents heatstroke by inhibition of high-mobility group box 1 protein in sera of rats. Shock. 2010;34(4):402-6.

9. Bouchama A, Kunzelmann C, Dehbi M, Kwaasi A, Eldali A, Zobairi F, et al. Recombinant activated protein $C$ attenuates endothelial injury and inhibits procoagulant microparticles release in baboon heatstroke. Arterioscler Thromb Vasc Biol. 2008;28(7):1318-25. 
10. Wu WS, Chou MT, Chao CM, Chang CK, Lin MT, Chang CP. Melatonin reduces acute lung inflammation, edema, and hemorrhage in heatstroke rats. Acta Pharmacol Sin. 2012;33(6):775-82.

11. Bouchama A, Kwaasi A, Dehbi M, Al Mohanna F, Eldali A, El-Sayed R, et al. Glucocorticoids do not protect against the lethal effects of experimental heatstroke in baboons. Shock. 2007;27(5):578-83.

12. Yeo TP. Heat stroke: a comprehensive review. AACN Clin Issues. 2004;15(2):280-93.

13. Chen ZC, Kuo JR, Huang YP, Lin MT. Mu-opioid receptor blockade protects against circulatory shock and cerebral ischemia during heatstroke. J Cardiovasc Pharmacol. 2005;46(6):754-60.

14. Wang Q, Yao YM, Wang WJ, Xian LM, Dong N, Xu S, et al. Effect of Xuebijing injection on renal high mobility group box-1 protein expression and acute kidney injury in rats after scald injury. Zhongguo Yi Xue Ke Xue Yuan Xue Bao. 2007;29(4):478-83.

15. Gao YL, Chai YF, Yao YM. Advancement in the research of mechanism of immune dysfunction in sepsis and the regulatory effects of Xuebijing injection. Zhonghua Shao Shang Za Zhi. 2013;29(2):162-5.

16. Chen $Y$, Tong $H$, Zhang $X$, Tang L, Pan Z, Liu Z, et al. Xuebijing injection alleviates liver injury by inhibiting secretory function of Kupffer cells in heat stroke rats. J Tradit Chin Med. 2013;33(2):243-9.

17. Jiang $M$, Zhou $M$, Han $Y$, Xing $L$, Zhao H, Dong $L$, et al. Identification of NF-kappaB Inhibitors in Xuebijing injection for sepsis treatment based on bioactivity-integrated UPLC-Q/TOF. J Ethnopharmacol. 2013;147(2):426-33.

18. Qi F, Liang ZX, She DY, Yan GT, Chen LA. A clinical study on the effects and mechanism of xuebijing injection in severe pneumonia patients. J Tradit Chin Med. 2011;31(1):46-9.

19. Guo SS, Gao YJ, Tian XC, Jin YH, Liu FZ, Cui XL. Effect of xuebijing oral effervescent tablet on endotoxin induced fever and disseminated intravascular coagulation rabbit model. Yao Xue Xue Bao. 2013;48(8):1241-6.

20. Ji J, Zhou F, Yue H, Song Q. Protective mechanism of Xuebijing injection against heat stroke in rats. Exp Ther Med. 2014;7(6):1745-51.

21. Dehbi M, Uzzaman T, Baturcam E, Eldali A, Ventura W, Bouchama A. Toll-like receptor 4 and high-mobility group box 1 are critical mediators of tissue injury and survival in a mouse model for heatstroke. PLoS One. 2012;7(9):e44100.

22. Bouchama A, Ollivier V, Roberts G, Al Mohanna F, de Prost D, Eldali A, et al. Experimental heatstroke in baboon: analysis of the systemic inflammatory response. Shock. 2005;24(4):332-5.

23. Lin MT, Liu HH, Yang YL. Involvement of interleukin-1 receptor mechanisms in development of arterial hypotension in rat heatstroke. Am J Physiol. 1997;273(4 Pt 2):H2072-2077.

24. Liu CC, Chien CH, Lin MT. Glucocorticoids reduce interleukin-1 concentration and result in neuroprotective effects in rat heatstroke. J Physiol. 2000;527(Pt 2):333-43.

25. Chen CC, Chen ZC, Lin MT, Hsu CC. Activated protein C improves heatstroke outcomes through restoration of normal hypothalamic and thermoregulatory function. Am J Med Sci. 2009;338(5):382-7.

26. Hagiwara S, Iwasaka H, Matsumoto S, Hasegawa A, Yasuda N, Noguchi T. In vivo and in vitro effects of the anticoagulant, thrombomodulin, on the inflammatory response in rodent models. Shock. 2010;33(3):282-8.

27. Hagiwara S, Iwasaka H, Shingu C, Matsumoto S, Uchida T, Noguchi T. High-dose antithrombin III prevents heat stroke by attenuating systemic inflammation in rats. Inflamm Res. 2010;59(7):511-8.

28. Hall DM, Buettner GR, Oberley LW, Xu L, Matthes RD, Gisolfi CV. Mechanisms of circulatory and intestinal barrier dysfunction during whole body hyperthermia. Am J Physiol Heart Circ Physiol. 2001;280(2):H509-521.

29. Tong H, Tang Y, Chen Y, Yuan F, Liu Z, Peng N, et al. HMGB1 activity inhibition alleviating liver injury in heatstroke. J Trauma Acute Care Surg. 2013;74(3):801-7.

30. Wang YB, Yao YM, Wang Q, Wang WJ. Effect of "Xuebijing injection" on expression of high mobility group box-1 protein and acute liver injury in rats with scald injury. Zhonghua Shao Shang Za Zhi. 2009;25(3):171-5.

31. Chavakis T, Kanczkowski W, Willenberg HS, Bornstein SR. Endothelial dysfunction: a critical determinant in inflammation-associated adrenal insufficiency? Eur J Clin Invest. 2011;41(8):917-9.

32. Girish KS, Paul M, Thushara RM, Hemshekhar M, Shanmuga Sundaram M, Rangappa KS, et al. Melatonin elevates apoptosis in human platelets via ROS mediated mitochondrial damage. Biochem Biophys Res Commun. 2013;438(1):198-204.
33. Hong S, Kim JY, Hwang J, Shin KS, Kang SJ. Heptachlor induced mitochondria-mediated cell death via impairing electron transport chain complex III. Biochem Biophys Res Commun. 2013;437(4):632-6.

34. Jayaprakasam B, Yang N, Wen MC, Wang R, Goldfarb J, Sampson H, et al. Constituents of the anti-asthma herbal formula ASHMITM synergistically inhibit IL-4 and IL-5 secretion by murine Th2 memory cells, and eotaxin by human lung fibroblasts in vitro. J Integr Med. 2013;11(3):195-205.

35. Karki R, Park CH, Kim DW. Extract of buckwheat sprouts scavenges oxidation and inhibits pro-inflammatory mediators in lipopolysaccharide-stimulated macrophages (RAW264.7). J Integr Med. 2013;11(4):246-52.

\section{Submit your next manuscript to BioMed Central and take full advantage of:}

- Convenient online submission

- Thorough peer review

- No space constraints or color figure charges

- Immediate publication on acceptance

- Inclusion in PubMed, CAS, Scopus and Google Scholar

- Research which is freely available for redistribution 